ARTÍCULO ORIGINAL

\title{
FACTORES ASOCIADOS A LA NO UTILIZACIÓN DE SERVICIOS DE SALUD EN PERSONAS LGBTI DE PERÚ
}

\author{
Luccio Romani(i)1,2,a, Katerine Ladera-Porta(i)3,a, Dante M. Quiñones-Laveriano(id),b, \\ Wagner Rios-Garcia@ ${ }^{5, a}$, Alejandro Juarez-Ubillus (1) 1,a, Jennifer Vilchez-Cornejo@ ${ }^{2, b}$ \\ 1 Facultad de Medicina Humana, Universidad de San Martín de Porres, Chiclayo, Perú. \\ 2 Red de Investigación en Salud Pública y Epidemiología, Lima, Perú. \\ 3 Facultad de Medicina Humana, Universidad Nacional de Ucayali, Ucayali, Perú. \\ ${ }^{4}$ Universidad Continental, Lima, Perú. \\ ${ }^{5}$ Facultad de Medicina Humana, Universidad Nacional San Luis Gonzaga, Ica, Perú. \\ a Estudiante de Medicina Humana; ${ }^{b}$ médico cirujano.
}

\section{RESUMEN}

Objetivo: Determinar los factores asociados a la no utilización de servicios de salud en una muestra de la población de lesbianas, gais, bisexuales, transgénero e intersexuales (LGBTI) de Perú. Materiales y métodos: Estudio transversal analítico, análisis de datos secundarios de la Primera Encuesta LGBTI de Perú. Se tomó como variable de interés a las personas que tuvieron alguna enfermedad durante los últimos doce meses y que tuvieron que recibir atención médica. Se calcularon razones de prevalencias crudas (RPc) y ajustadas (RPa), con intervalos de confianza al 95\% (IC 95\%), usando regresiones de Poisson con varianza robusta. Se desarrollaron tres modelos, ajustados a variables agrupadas en correspondencia con la orientación sexual, identidad de género e intersexualidad, respectivamente. Resultados: El 55,4\% fueron registrados como varones al nacer, la mediana de la edad fue 25 años (Rango intercuartil: 21-30). El 16\% manifestó no haber buscado atención médica. Los tres modelos presentaron una asociación negativa respecto de padecer una enfermedad crónica, enfermedad infectocontagiosa, enfermedad mental y en quienes expresaban su orientación abiertamente. El no ser respetados por el género con el que se identificaban estuvo relacionado a no usar los servicios en el modelo 3. Los modelos 1 y 3 , incluyeron una asociación positiva con no ser respetados con el género que se identifica. Conclusión: Padecer de alguna enfermedad mental, una enfermedad infectocontagiosa y una enfermedad crónica además de no ser tratado con respeto según el género con el que se identifica son factores asociados a la no utilización de los servicios de salud.

Palabras clave: Minorías Sexuales y de Género; Servicios de Salud; Disparidades en la Atención de Salud; Perú (Fuente: DeCS BIREME).

\section{FACTORS ASSOCIATED WITH THE NON-USE OF HEALTH SERVICES IN LGBTI PEOPLE FROM PERU}

Citar como: Romani L, Ladera-Porta K, Quiñones-Laveriano DM, Rios-Garcia W, Juarez-Ubillus A, Vilchez-Cornejo J. Factores asociados a la no utilización de servicios de salud en personas LGBTI de Perú. Rev Peru Med Exp Salud Publica. 2021;38(2):240-7. doi: https://doi. org/10.17843/rpmesp.2021.382.6149.

Correspondencia: Luccio A. Romaní Ojeda; Avenida Víctor Raúl Haya de la Torre 565, Chiclayo, Lambayeque, Perú; luccioromani@gmail.com

Recibido: 08/07/2020 Aprobado: 03/02/2021 En línea: 22/06/2021

\section{ABSTRACT}

Objective: To determine the associated factor with the non-use of health services in a sample of the lesbian, gay, bisexual, transgender, and intersex population of Peru. Materials and methods: Analytical cross-sectional study, analysis of secondary data from the First LGBTI Survey of Peru. Those who suffered from a medical condition during the last twelve months who had to receive medical attention were considered a variable of interest. Crude prevalence ratios (PRc) and adjusted (PRa), with 95\% confidence intervals (95\% CI) were calculated using Poisson regressions with robust variance. Three models were developed, adjusted to variables grouped according to sexual orientation, gender identity, and intersexuality. Results: $55.4 \%$ were male at birth, the median age was 25 years (IR: $21-30$ ). 16\% stated that they had not sought medical attention. The three models presented a negative association in having suffered a chronic disease, infectious, contagious diseases, and mental illness and those who expressed their orientation openly. Not being respected for the gender they identified was related to not using the services in model 3 . Models 1 and 3 included a positive association with not being respected with the gender identified. Conclusion: Suffering from a mental illness, an infectious contagious disease, a chronic disease, and not being treated with respect according to their gender identity are factors associated with the non-use of health services.

Keywords: Sexual and gender minorities; Health Services, Healthcare Disparities, Peru (Source: MeSH NLM). 


\section{INTRODUCCIÓN}

En las últimas décadas, la conciencia pública e interés científico del abordaje de los problemas que sufren la comunidad de lesbianas, gais, bisexuales, transgénero e intersexuales (LGBTI) ha ido en aumento ${ }^{(1)}$. Esto se debe, principalmente, a la heteronormatividad o el heterosexismo, que genera violencia y discriminación, y se agrava por la estigmatización de la orientación sexual ${ }^{(2,3)}$.

Las brechas existentes con respecto al acceso a los servicios de salud en personas del colectivo LGBTI se centran en la discriminación estructural y la violencia institucionalizada que padece esta comunidad, lo que genera múltiples disparidades a la hora de recibir atención médica. Este factor está fuertemente asociado con no acudir a los servicios de salud debido al estigma internalizado y con haber sufrido discriminación por parte de los trabajadores de salud o pacientes ${ }^{(3,4)}$. Esto genera un ambiente de desconfianza y falta de comunicación en el sistema de salud por parte de la comunidad LGBTI, aislamiento crónico, aplazamiento o negación a recibir atención médica, incluso cuando sus dolencias pueden poner en gran riesgo su salud y deben ser atendidos muchas veces por emergencia ${ }^{(5)}$.

Un estudio realizado en Estados Unidos demostró que el 18\% de los adultos LGBTI reportó haber evadido la búsqueda de atención médica para ellos mismos o para sus familiares, esto por la discriminación anticipada, mientras que el $16 \%$ informó discriminación en servicios de salud ${ }^{(4)}$. En las personas transgénero, un estudio observó que tenían más probabilidades de retrasar la atención médica en comparación con los participantes cisgénero, debido a que anticipan la discriminación ${ }^{(5)}$. Asimismo, en mujeres transexuales, el $41 \%$ evitó buscar atención médica debido principalmente al estigma y la discriminación ${ }^{(3)}$.

A esto se le suma que algunas políticas de salud, como la de prevención de $\mathrm{VIH} /$ sida, dirigidas específicamente a una subpoblación (por ejemplo, quienes se dedican al trabajo sexual), se implementen con una tácita señalización y estigmatización de estas personas, lo que aumenta el rechazo de la asistencia por el sistema de salud ${ }^{(6)}$. Así, ante la amenaza de sentirse que el estar presentes o gozar de algún beneficio no es lo correcto para ellos, pueden autoexcluirse de estos servicios $^{(7)}$.

En Perú, se reportó que el 26\% de peruanos miembros de la comunidad LGBTI percibía que el acceso a la salud sigue siendo vulnerado con múltiples brechas respecto a su acceso. Ayacucho y Piura son las regiones con una mayor tasa de vulnerabilidad (39\% y $35 \%$ respectivamente) ${ }^{(8)}$. La discriminación y la violencia institucionalizadas generan desigualdades a la hora de recibir atención médica y constituyen las brechas institucionales descritas en diversos

\section{MENSAJES CLAVE}

Motivación para realizar el estudio: Uno de los principales problemas de la comunidad LGBTI son las brechas en el acceso a la salud. En Perú, la discriminación y la violencia institucionalizada generan desigualdades a la hora de recibir atención médica.

Principales hallazgos: Los factores asociados a la no utilización de servicios de salud fueron tener una enfermedad mental y no ser tratado por el género con el que se identifica.

Implicancias: La creación de protocolos de atención integral con enfoque a las personas LGBTI, permitiría mejorar el trato digno y no discriminatorio a esta población.

contextos ${ }^{(9)}$; aún no se tiene información de las condiciones que llevan a una persona LGBTI a no utilizar los servicios de salud en caso de enfermedad.

La presente investigación tiene como objetivo determinar los factores asociados a la no utilización de los servicios de salud en una muestra de la población LGBTI de Perú. Esto ayudará a comprender las razones por las cuales esta población renuncia a sus derechos de salud. Asimismo, la elaboración de protocolos de atención integral y la promoción de futuras investigaciones enfocadas al colectivo LGBTI permitiría comprender el trato que se le brinda, teniendo como misión establecer un punto inicial de reflexión respecto a una ansiada atención de salud integral en las poblaciones más vulnerables, como la comunidad LGBTI.

\section{MATERIALES Y MÉTODOS}

\section{Diseño y área del estudio}

Se realizó un estudio transversal analítico, de datos secundarios de la Primera encuesta virtual para personas LGBTI, efectuada por el Instituto Nacional de Estadística e Informática (INEI) en el 2017. Esta encuesta es de carácter exploratorio, no probabilístico, por cuanto no se dispuso de información preliminar acerca del tamaño de la población.

La encuesta se realizó entre el 17 de mayo y el 17 de agosto del 2017 a través de un cuestionario en línea difundido en la página web del INEI en las redes sociales y en otros medios electrónicos. Contando con el apoyo de diversas organizaciones LGBTI para alcanzar a encuestar a la población objetivo e intentando eliminar las respuestas sesgadas por personas no LGBTI. La cobertura de la encuesta abarcó todo el territorio nacional peruano, incluyendo zonas urbanas y rurales $^{(10)}$. 


\section{Población y muestra}

La encuesta incluyó a personas de 18 años o más, residentes en el territorio peruano que se identificaron como parte del colectivo LGBTI o que, sin identificarse con dichas categorías no se adscriben a las definiciones binarias o rígidas de lo masculino o femenino, tuvieron acceso al cuestionario electrónico. La población inicial de la encuesta fue de 12027 participantes de todo el país.

Se excluyó a los participantes que no respondieron a las preguntas que les permitiera identificarse o definirse como parte de colectivo, correspondientes a las preguntas 112, 113,114 y 120 del cuestionario ${ }^{(10)}$, las cuales abordaban el sexo asignado al nacer, orientación sexual, identidad de género y su intersexualidad, respectivamente. Además, se excluyó a quienes no respondieron las preguntas 105 y 106, correspondientes a la variable de interés que delimita a quienes han tenido un problema de salud durante los últimos doce meses y han acudido a algún establecimiento de salud para recibir una atención, respectivamente. Finalmente, solo se consideró a los miembros de las comunidades LGBTI que a pesar de haber tenido la necesidad de utilizar los servicios de salud no lo hicieron por diversos motivos. La población final fue de 5386 participantes.

\section{Variables e instrumentos}

El cuestionario electrónico estuvo constituido por 71 preguntas, que incluían datos generales que consideraban características sociodemográficas como edad, sexo asignado al nacer, educación, salud, ocupación, identidad, cuerpo y sexualidad, entorno familiar, discapacidad, etnicidad (36 preguntas); discriminación y violencia (11 preguntas); conocimientos de los derechos de las personas LGBTI (3 preguntas); participación ciudadana (2 preguntas); percepción sobre la condición de la persona LGBTI (4 preguntas) y datos de la vivienda y del hogar (5 preguntas).

Las variables incluidas en el estudio se determinaron a partir de una revisión teórica previa, acerca de las barreras de las minorías sexuales para el acceso a servicios de salud ${ }^{(11)}$, en la que se consideran aspectos relacionados a la orientación sexual, identidad de género, condición de intersexualidad; además aspectos socioeconómicos, geográficos, de demanda y oferta que influyen en el acceso a los servicios de salud ${ }^{(12)}$. Para determinar la variable dependiente, se seleccionó primero a las personas que habían reportado tener alguna enfermedad (pregunta 105: «En los últimos 12 meses, usted tuvo algún problema de salud como...»). Luego, se cruzó con la respuesta "No busqué atención», alternativa de respuesta correspondiente a la pregunta 106 ( ¿ $\mathrm{A}$ dónde acudió para atenderse por esta enfermedad?»).

Asimismo, se consideraron las preguntas que abarcaban las variables de edad, sexo registrado al nacer, lugar de residencia, etnicidad, situación sentimental, nivel educativo, tener un seguro de salud, antecedentes de enfermedades crónicas no trans- misibles (asma, bronquitis crónica, enfisema, hipertensión arterial, diabetes mellitus) en los últimos doce meses, antecedentes de enfermedades infectocontagiosas (tuberculosis, infecciones de transmisión sexual, VIH/sida) o de salud mental (ansiedad, depresión), los motivos para no utilizar los servicios de salud, haber sufrido algún tipo de discriminación por el personal de salud, haber sido obligado a someterse a pruebas de ITS o VIH. Además, se incluyeron variables respecto a la orientación sexual, diversidad de género y características sexuales, orientación sexual, identidad de género, intersexualidad, si expresa abiertamente su orientación sexual, si algún familiar conoce sobre su orientación sexual o identidad de género. Las definiciones de los términos usados en las variables correspondientes a la diversidad sexual, de género y características sexuales se encuentran en el glosario adjunto como material suplementario.

\section{Procedimiento y análisis estadístico}

Se descargaron los módulos de datos desde la página web del INEI para analizar los datos. Posteriormente, se extrajeron los datos necesarios del módulo colgado en SPSS v.25, se los exportó a un archivo de Microsoft Excel 2013 y se mantuvieron solo las variables de interés previamente delimitadas y corroboradas por los autores.

Se analizaron los datos en el paquete estadístico Stata versión 15 (StataCorp, College Station, Texas, EE. UU.), en dónde se presentaron las variables cualitativas en frecuencias y porcentajes; las variables cuantitativas se presentaron en medianas y rangos intercuartílicos, después de evaluar su distribución.

Se realizó una regresión lineal simple a todas las variables y se calcularon los factores de inflación de la varianza para evaluar multicolinealidad entre las variables independientes. Se encontró que las variables identidad de género, orientación sexual e intersexualidad presentaban multicolinealidad. Por ello, y debido a la importancia de estas variables en el estudio, se desarrollaron tres modelos con la intención de incluir estas variables en el mismo modelo de análisis ajustado. El análisis multivariado incluyó en cada modelo las variables con un $p<0,20$ en el análisis bivariado: edad, lugar de residencia, nivel educativo, seguro de salud, antecedentes de ITS, antecedentes de enfermedades crónicas no transmisibles, antecedentes de enfermedades de salud mental, expresa sin temor su orientación sexual, relación de pareja, obligado a realizarse pruebas de ITS, no respetaron el género con el que se identifica, tuvo que cambiarse de apariencia y sexo asignado al nacer.

En cada modelo se calcularon razones de prevalencias crudas (RPc) y ajustadas ( $\mathrm{RPa})$, así como sus intervalos de confianza al 95\% (IC 95\%), usando regresiones de Poisson con varianza robusta. Se consideró una asociación estadísticamente significativa a las razones de prevalencias con un IC 95\% que no incluyeran al uno. 


\section{Consideraciones éticas}

El protocolo del presente trabajo se encuentra en el Registro Nacional de Proyectos de Investigaciones en Salud (PRISA) del INS (Código de registro: EI0000000660). Además, se tuvieron en cuenta las consideraciones éticas expresadas en la declaración de Helsinki, se extrajeron de la base de datos solo los considerados relevantes para el estudio y se ignoraron los que nos permitieran reconocer la identidad de los individuos. Los datos usados en la presente investigación son de dominio público y no contienen información que permita la identificación de algún individuo.

\section{RESULTADOS}

Se contó con una población final de 5386 personas, luego de excluir al 6,4\% (771) por no identificarse como parte del colectivo (heterosexuales, no intersexuales, no transgénero) y al 48,8\% (5870) por no responder a la pregunta si presentaron alguna enfermedad en los últimos doce meses y finalmente el $0,1 \%$ (11) de registros que fueron eliminados debido a que contaron con información incompleta y no concordante cuyo contenido era ofensivo y/u obsceno al momento de ser evaluado por los autores, estos datos se encuentran en detalle en un flujograma (Figura 1).

El 55,4\% (2984) fueron registrados de sexo masculino al nacer, la mediana de la edad corresponde a 25 años, los rangos intercuartílicos 21 a 30. El 44,2\% (2379) reportó ser gay, el 5,9\% (316) ser transexual y solo el 4,4\% (233) indicó ser intersexual. Asimismo, el 57,1\% (3058) de los participantes manifestó contar con estudios universitarios y el 52,2\%
(2777) manifestó no tener pareja. Las demás características generales se encuentran resumidas en la Tabla 1.

Respecto al ámbito de salud, el 29,7\% (1600) manifestó no tener seguro. Durante los últimos doce meses, el $49,4 \%$ (2658) manifestó haber padecido alguna enfermedad mental correspondiente a trastornos de adaptación, como ansiedad o depresión. Sin embargo, el 16\% (862) de los participantes manifestó que no habían buscado atención médica, siendo la falta de dinero el motivo más frecuente (31,3\%). La distribución geográfica de la no utilización de los servicios de salud se encuentra expresado en la Figura 2.

Acerca de la no utilización de los servicios de salud, respecto al análisis multivariado, se presentó una asociación negativa en los tres modelos, con tener estudios de posgrado, haber padecido de alguna enfermedad crónica no transmisible, haber padecido de alguna infección de transmisión sexual, expresar abiertamente su orientación sexual o identidad de género; además, el modelo 3 incluyó la asociación negativa con residir en Lima. Asimismo, se encontró en los tres modelos una asociación positiva con haber presentado algún problema de salud mental correspondiente a un trastorno adaptativo; por otro lado, en los modelos 1 y 3 se incluyó una asociación positiva con no haber sido respetado con el género que se identifica (Tabla 2).

\section{DISCUSIÓN}

En la presente investigación se encontró que la limitación socioeconómica y la falta de confianza en el personal de salud

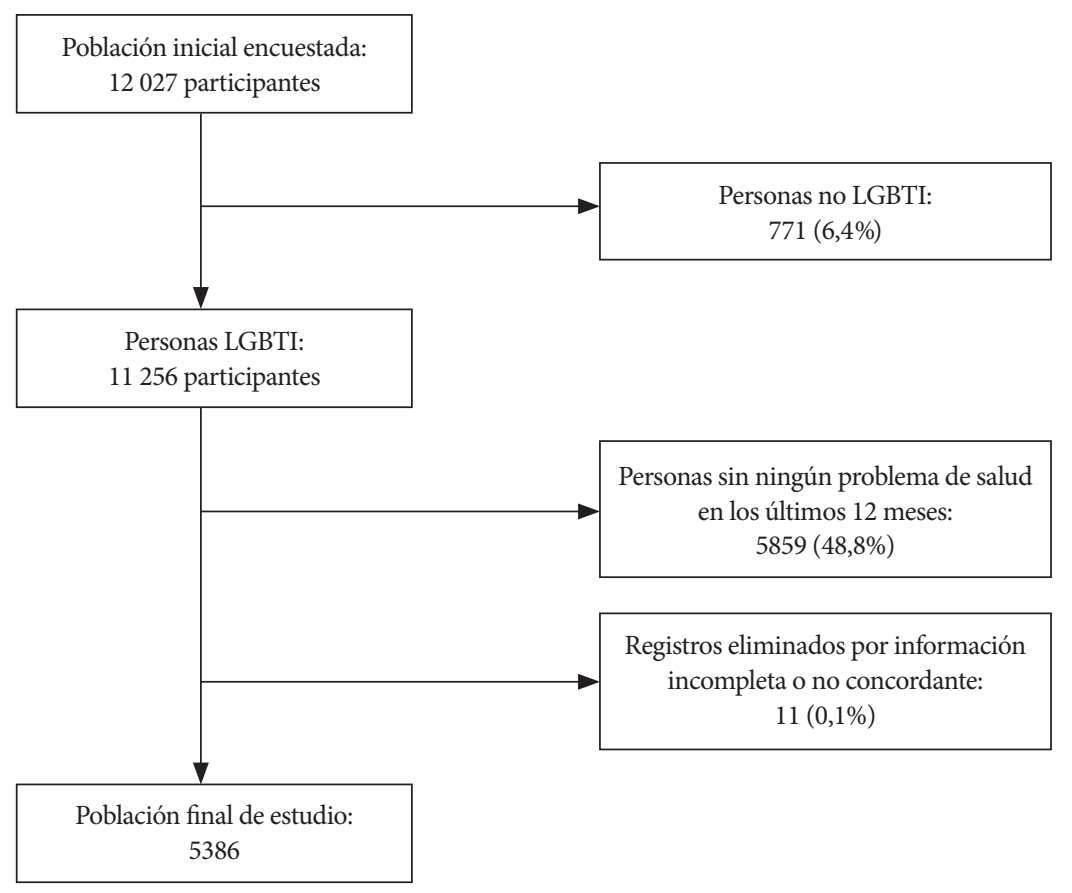

Figura 1. Flujograma de la selección de la población estudiada. 
Tabla 1. Características generales de la población LGBTI estudiada.

\begin{tabular}{|c|c|c|}
\hline Característica & $\mathbf{n}$ & $\%$ \\
\hline Edad & \multicolumn{2}{|c|}{$25(21-30)^{\mathrm{a}}$} \\
\hline \multicolumn{3}{|l|}{ Sexo registrado al nacer } \\
\hline Femenino & 2400 & 44,6 \\
\hline Masculino & 2984 & 55,4 \\
\hline \multicolumn{3}{|l|}{ Grado de instrucción } \\
\hline Sin estudios superiores & 751 & 14,0 \\
\hline Con estudios técnicos & 1089 & 20,3 \\
\hline Estudios universitarios & 3058 & 57,1 \\
\hline Estudios de posgrado & 461 & 8,6 \\
\hline \multicolumn{3}{|l|}{ Pareja } \\
\hline Sin pareja & 2777 & 52,2 \\
\hline Con pareja, sin convivencia & 1671 & 31,4 \\
\hline Con pareja, en convivencia & 764 & 14,4 \\
\hline Con más de una pareja & 105 & 2,0 \\
\hline \multicolumn{3}{|l|}{ Orientación sexual } \\
\hline Heterosexual & 119 & 2,2 \\
\hline Homosexual, gay & 2379 & 44,2 \\
\hline Homosexual, lesbiana & 989 & 18,4 \\
\hline Bisexual & 1366 & 25,4 \\
\hline Otro $^{b}$ & 530 & 9,8 \\
\hline \multicolumn{3}{|l|}{ Intersexual } \\
\hline No & 4477 & 84,2 \\
\hline Sí & 233 & 4,38 \\
\hline No sabe & 607 & 11,42 \\
\hline \multicolumn{3}{|l|}{ Identidad de género } \\
\hline Persona transgénero $^{c}$ & 316 & 5,9 \\
\hline Persona cisgénero & 4540 & 85,2 \\
\hline Persona de queer género ${ }^{d}$ & 474 & 8,9 \\
\hline
\end{tabular}

a Mediana y rangos intercuartílicos; ${ }^{\mathrm{b}}$ pansexual, asexual, demisexual; ' transexual, transgénero, travesti; ${ }^{\mathrm{d}}$ personas de género no binario.

fueron los principales motivos para no utilizar los servicios de salud, lo cual tendría relación con lo reportado en estudios previos; donde, en comparación con los heterosexuales, las minorías sexuales tienden a retrasar la atención debido al costo, experiencias negativas previas en la atención médica, no poder obtener citas y evitar molestar a un proveedor ${ }^{(13)}$. Uno de cada seis adultos LGBTI indicaron que evitaron buscar atención médica debido a la discriminación anticipada y experimentada en los centros de atención médica ${ }^{(4)}$.

Se encontró que tener educación superior de posgrado disminuyó la prevalencia de no utilización de servicios de salud. Si bien los estudios específicos de este determinante en la población LGBTI son escasos, se sabe que el nivel educativo influye en el acceso adecuado a la salud ${ }^{(14)}$. Un nivel de estudios superior se relaciona con un mejor nivel socioeconómico y menores barreras para acceder a los servicios de salud ${ }^{(15)}$. Por otro lado, residir en Lima disminuyó la frecuencia de no utilización de servicios de salud. Esto po-

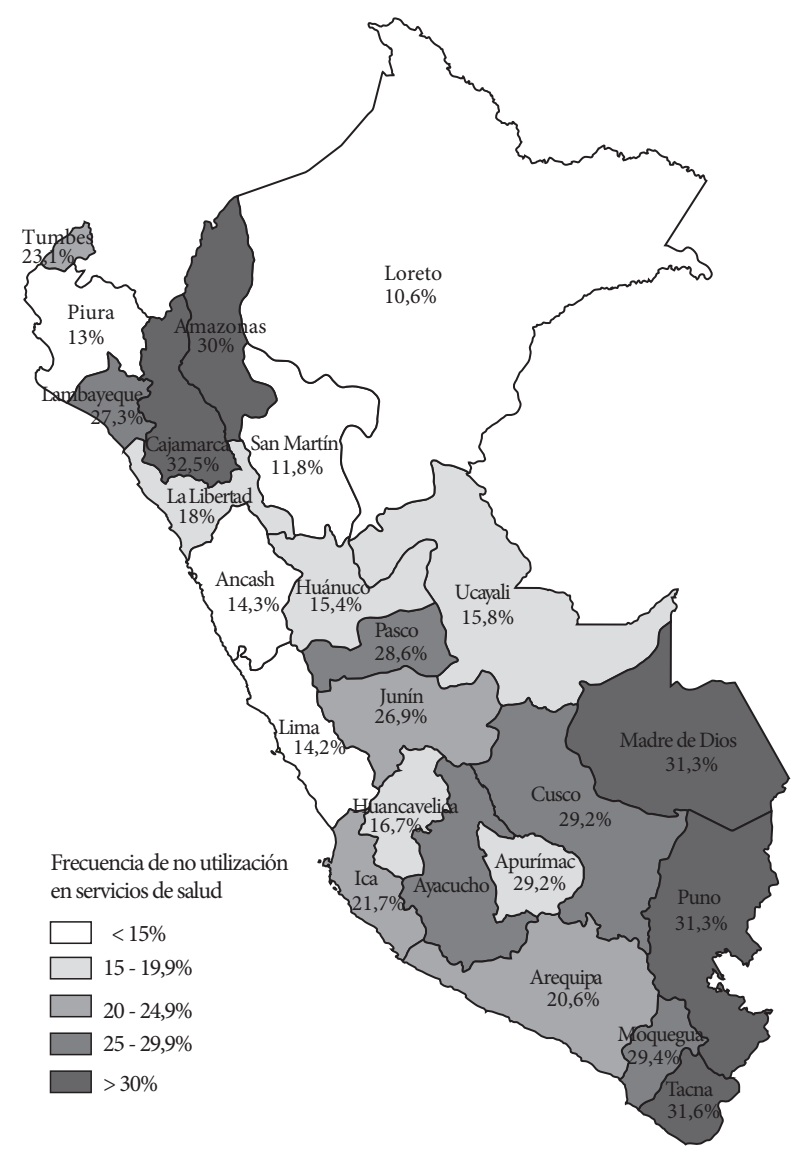

Figura 2. Frecuencia de no utilización de servicios de salud por ubicación geográfica.

dría deberse a que, a diferencia de otras ciudades de Perú, en Lima se han implementado de manera progresiva servicios de salud con enfoque de derechos orientados a la integración y acogimiento de la población LGBTI ${ }^{(16)}$. Queda el desafío pendiente de su implementación y fortalecimiento a nivel nacional ${ }^{(17)}$.

También se encontró que la prevalencia de no utilización de los servicios de salud fue menor en quienes tenían un seguro de salud. El no tener un seguro de salud podría implicar la necesidad de realizar un pago por la atención médica, lo cual constituiría una barrera económica. Esto concuerda con lo reportado en Estados Unidos, donde la minoría sexual tuvo mayor frecuencia de retraso de atención médica debido a los $\operatorname{costos}^{(18)}$. Si bien la mayoría de la población reportó estar afiliada a un seguro de salud, existen grupos, como la población transgénero, que ven difícil su afiliación debido a que muchas veces no utilizan su nombre legal por no coincidir con su identidad de género ${ }^{(19)} \mathrm{o}$ porque los servicios ofrecidos no cubren adecuadamente sus necesidades ${ }^{(20)}$.

Por otro lado, casi la mitad de la muestra estudiada reportó haber sufrido algún problema de salud mental en los últimos doce meses. Este hallazgo es similar al reportado en diversos estudios nacionales e internacionales ${ }^{(21,22)}$, donde el estrés de las minorías, la estigmatización y la discriminación afectan 
Tabla 2. Análisis bivariado y multivariado entre los factores asociados a la no utilización de servicios de salud en personas LGBTI en Perú.

\begin{tabular}{|c|c|c|c|c|c|c|c|c|}
\hline \multirow{3}{*}{ Variable } & \multicolumn{8}{|c|}{ No utilización de servicios de salud } \\
\hline & \multicolumn{2}{|c|}{ Modelo crudo } & \multicolumn{2}{|c|}{ Modelo ajustado 1} & \multicolumn{2}{|c|}{ Modelo ajustado 2} & \multicolumn{2}{|c|}{ Modelo ajustado 3} \\
\hline & RPc (IC 95\%) & Valor de p & $\mathrm{RPa}(\mathrm{IC} 95 \%)$ & Valor de p & RPa (IC 95\%) & Valor de $\mathrm{p}$ & $\mathrm{RPa}(\mathrm{IC} 95 \%)$ & Valor de $\mathrm{p}$ \\
\hline Edad & $0,94(0,93-0,95)$ & $<0,001$ & $0,99(0,98-1,00)$ & 0,118 & $0,99(0,98-1,00)$ & 0,115 & $0,99(0,98-1,00)$ & 0,081 \\
\hline \multicolumn{9}{|l|}{ Sexo registrado al nacer } \\
\hline Femenino & Referencia & & Referencia & & Referencia & & Referencia & \\
\hline Masculino & $0,64(0,56-0,73)$ & $<0,001$ & $1,17(0,95-1,45)$ & 0,129 & $1,19(1,04-1,37)$ & 0,013 & $1,19(1,04-1,36)$ & 0,013 \\
\hline No & Referencia & & Referencia & & Referencia & & Referencia & \\
\hline Sí & $0,70(0,61-0,81)$ & $<0,001$ & $0,80(0,70-0,93)$ & 0,003 & $0,80(0,70-0,93)$ & 0,003 & $0,80(0,64-0,93)$ & 0,003 \\
\hline \multicolumn{9}{|c|}{ Le obligaron a someterse a pruebas de ITS o VIH } \\
\hline No & Referencia & & Referencia & & Referencia & & Referencia & \\
\hline Sí & $0,60(0,44-0,82)$ & $<0,001$ & $0,78(0,60-1,03)$ & 0,078 & $0,78(0,60-1,03)$ & 0,078 & $0,80(0,61-1,05)$ & 0,610 \\
\hline \multicolumn{9}{|l|}{ Nivel de estudios alcanzado } \\
\hline Con estudios técnicos & $0,74(0,61-0,91)$ & 0,004 & $1,03(0,81-1,25)$ & 0,972 & $1,01(0,81-1,26)$ & 0,943 & $0,99(0,80-1,24)$ & 0,956 \\
\hline Estudios universitarios & $0,82(0,69-0,96)$ & 0,017 & $0,89(0,74-1,07)$ & 0,208 & $0,89(0,74-1,07)$ & 0,198 & $0,89(0,74-1,06)$ & 0,197 \\
\hline Estudios de posgrado & $0,31(0,21-0,46)$ & $<0,001$ & $0,50(0,33-0,76)$ & 0,001 & $0,49(0,32-0,75)$ & 0,001 & $0,49(0,34-0,75)$ & 0,001 \\
\hline \multicolumn{9}{|l|}{ Relación de pareja } \\
\hline Sin pareja & Referencia & & Referencia & & Referencia & & Referencia & \\
\hline Con pareja sin convivencia & $0,90(0,78-1,05)$ & 0,211 & $0,95(0,82-1,10)$ & 0,486 & $0,96(0,83-1,11)$ & 0,563 & $0,96(0,87-1,11)$ & 0,577 \\
\hline Con pareja en convivencia & $0,62(0,49-0,78)$ & $<0,001$ & $0,95(0,76-1,18)$ & 0,647 & $0,93(0,74-1,17)$ & 0,547 & $0,95(0,76-1,19)$ & 0,662 \\
\hline Más de una pareja & $0,65(0,36-1,15)$ & 0,141 & $1,17(0,62-2,18)$ & 0,630 & $1,36(0,75-2,45)$ & 0,314 & $1,20(0,65-2,22)$ & 0,560 \\
\hline \multicolumn{9}{|l|}{ Lugar de residencia } \\
\hline Provincia & Referencia & & Referencia & & Referencia & & Referencia & \\
\hline Lima & $0,84(0,73-0,97)$ & 0,021 & $0,98(0,95-1,02)$ & 0,378 & $0,98(0,95-1,02)$ & 0,399 & $0,98(0,95-1,02)$ & $<0,001$ \\
\hline \multicolumn{9}{|l|}{ Seguro de salud } \\
\hline No & Referencia & & Referencia & & Referencia & & Referencia & \\
\hline Sí & $0,51(0,44-0,58)$ & $<0,001$ & $0,75(0,66-0,85)$ & $<0,001$ & $0,75(0,66-0,86)$ & $<0,001$ & $0,75(0,66-0,85)$ & $<0,001$ \\
\hline Sí & $0,26(0,21-0,33)$ & $<0,001$ & $0,72(0,33-0,54)$ & $<0,001$ & $0,42(0,33-054)$ & $<0,001$ & $0,41(0,33-0,54)$ & $<0,001$ \\
\hline \multicolumn{9}{|c|}{ Antecedentes de enfermedades infectocontagiosas } \\
\hline No & Referencia & & Referencia & & Referencia & & Referencia & \\
\hline Sí & $0,10(0,07-0,15)$ & $<0,001$ & $0,18(0,11-0,29)$ & $<0,001$ & $0,18(0,12-0,29)$ & $<0,001$ & $0,18(0,11-0,29)$ & $<0,001$ \\
\hline \multicolumn{9}{|c|}{ Antecedentes de problemas de salud mental } \\
\hline No & Referencia & & Referencia & & Referencia & & Referencia & \\
\hline Sí & $8,48(6,84-10,52)$ & $<0,001$ & $4,78(3,71-6,15)$ & $<0,001$ & $4,85(3,77-6,25)$ & $<0,001$ & $4,74(3,68-6,10)$ & $<0,001$ \\
\hline Respeto por su identidad de gen & & & & & & & & \\
\hline No & Referencia & & Referencia & & Referencia & & Referencia & \\
\hline Sí & $1,25(1,05-1,50)$ & 0,014 & $1,26(1,06-1,50)$ & 0,010 & $1,29(0,93-1,23)$ & 0,333 & $1,26(1,06-1,49)$ & 0,008 \\
\hline Cambio de apariencia & & & & & & & & \\
\hline No & Referencia & & Referencia & & Referencia & & Referencia & \\
\hline Sí & $1,34(1,16-1,56)$ & $<0,001$ & $1,08(0,94-1,24)$ & 0,288 & $1,08(0,93-1,24)$ & 0,333 & $1,08(0,94-1,24)$ & 0,285 \\
\hline Orientación sexual & & & & & & & & \\
\hline Heterosexual & Referencia & & Referencia & & - & - & - & - \\
\hline Homosexual, gay & $0,67(0,45-0,99)$ & 0,049 & $1,11(0,72-1,72)$ & 0,636 & - & - & - & - \\
\hline Homosexual, lesbiana & $0,89(0,59-1,35)$ & 0,595 & $1,08(0,70-1,69)$ & 0,722 & - & - & - & - \\
\hline Bisexual & $1,17(0,78-1,75)$ & 0,444 & $1,14(0,75-1,75)$ & 0,540 & - & - & - & - \\
\hline Otros $^{\mathrm{a}}$ & $1,31(0,87-1,99)$ & 0,199 & $1,09(0,70-1,71)$ & 0,693 & - & - & - & - \\
\hline Identidad de género & & & & & & & & \\
\hline Cisgénero & Referencia & & - & - & Referencia & & - & - \\
\hline Transgénero & $1,17(0,801,71)$ & 0,409 & - & - & $0,91(0,69-1,20)$ & 0,515 & - & - \\
\hline Queer género & $1,30(1,051,61)$ & 0,014 & - & - & $1,07(0,88-1,12)$ & 0,496 & - & - \\
\hline Intersexualidad & & & & & & & & \\
\hline No & Referencia & & - & - & - & - & Referencia & \\
\hline Sí & $0,57(0,38-, 086)$ & 0,007 & - & - & - & - & $0,64(0,41-0,99)$ & 0,045 \\
\hline No sabe & $1,25(1,05-1,48)$ & 0,013 & - & - & - & - & $1,14(0,94-1,37)$ & 0,175 \\
\hline
\end{tabular}

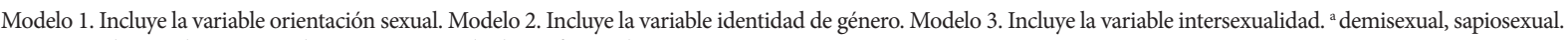
RPa: razón de prevalencia ajustada; IC 95\%: intervalo de confianza al 95\%. 
negativamente su salud mental ${ }^{(23)}$, particularmente en entornos intolerantes como podría ser la sociedad peruana ${ }^{(24)}$. Esto hace que la atención adecuada de los problemas de salud mental de las personas LGBTI sea una prioridad; sin embargo, a diferencia de los problemas de salud como enfermedades crónicas no transmisibles o ITS, las enfermedades mentales aumentaron la prevalencia de no utilización de los servicios de salud. Un estudio canadiense reporta que las principales barreras de las minorías sexuales para atenderse por salud mental fueron la imposibilidad de pagar, una cobertura de seguro insuficiente, preferían «esperar» a que los problemas desaparezcan, la incomodidad de hablar de emociones y vergüenza ${ }^{(25)}$. De estas barreras, es probable que las dos últimas tengan connotaciones particulares en el contexto peruano, donde el personal de salud no está capacitado para atender a personas LGBTI y, peor aún, tendrían actitudes negativas frente a ellas ${ }^{(26)}$. Esto llevaría no solo a la inadecuada atención de la salud mental, sino a la exposición de actitudes negativas o violentas. Dados los desafíos específicos de personas LGBTI, como problemas en el desarrollo de una identidad, relaciones de pareja, problemas en la crianza, problemas relacionados a sus familias, entre otros, se necesita incluir pautas en todo el país para atender su salud mental, y disminuir el heterosexismo y el sesgo en la atención médica ${ }^{(26,27)}$.

Se observó que expresar sin temor su orientación sexual e identidad de género aumentó la frecuencia de no utilización de servicios de salud. Este empoderamiento del paciente podría facilitar la atención médica adecuada para sus problemas de salud, sin embargo, debe acompañarse de un entorno que facilite este escenario. Así pues, un estudio en Estados Unidos encontró que, en aquellos estados con leyes contra la discriminación de las minorías sexuales, las mujeres de minorías sexuales fueron más propensas a declarar su identidad de género u orientación sexual a su proveedor de atención de la salud, lo cual mejoró la atención médica y la satisfacción de la paciente ${ }^{(28)}$.

Por otro lado, también se encontró que, en los modelos para identidad de género e intersexualidad, no respetar el género con el que se identifica el paciente aumentó la prevalencia de no utilización de los servicios de salud. Esto concuerda con un estudio realizado en Estados Unidos, donde se encontró que las personas transgénero tenían una alta proporción de retrasar o no buscar atención médica debido a la discriminación del sistema de salud ${ }^{(29)}$. También se ha observado que los proveedores de salud, especialmente los heterosexuales y varones, podrían tener actitudes negativas frente a pacientes LGBTI, lo cual es una manifestación de la discriminación sistémica al que nuestra sociedad los somete ${ }^{(26)}$.

Se ha encontrado que reconocerse como intersexual disminuyó la frecuencia de no utilización de los servicios de salud. Esto puede deberse a que las personas intersexuales necesitan de una atención médica adecuada que les permita entender las características físicas de la intersexualidad y la identidad de género no binaria, hasta que el paciente esté en condiciones de identificarse con algún género y algún sexo, si así lo desea ${ }^{(30)}$. La investigación de los problemas que afectan a las personas intersexuales es aún escasa, por lo que estos hallazgos deben ser contextualizados en el marco de estudios específicos que puedan llevar a mejores políticas, educación, atención de salud y responsabilidad ética por parte de nuestro sistema sanitario.

Es importante la información sobre las necesidades de las personas LGBTI en general y con énfasis en los futuros profesionales de salud, con información útil y confiable, que incluya en la atención integral de la comunidad orientada a personas LGBTI. Asimismo, el Estado, en su deber de asegurar un servicio a la salud universal respetando los derechos de todos los pobladores, debería abordar las disparidades del acceso y utilización de servicios de salud que actualmente son fragmentados, siendo este colectivo uno de los más vulnerables. La creación de protocolos de atención integral con enfoque a las personas LGBTI permitiría sensibilizar y educar al personal de salud.

La investigación contó con diversas limitaciones. La encuesta, al tener un carácter exploratorio no probabilístico, no dispuso de información previa sobre el tamaño de esta población para poder seleccionar una muestra. En vista de ello, los resultados obtenidos están referidos a la población que participó en la encuesta y no pueden ser extrapolados al total de la población LGBTI de Perú. Debido a que la encuesta se realizó de manera virtual, el acceso estuvo limitado a las personas LGBTI que tenían internet. Algunas de las preguntas corresponden a eventos sucedidos hasta con doce meses de anticipación por lo que podría existir sesgo de memoria en el llenado de la encuesta, a pesar de ello las variables incluidas delimitan de manera detallada y específica cada una de las variables de interés. Del mismo modo, las respuestas de la encuesta al ser autorreportada puede constituir un sesgo de información. Finalmente, el diseño de la encuesta impidió realizar un estudio de casos y controles por lo que no existe una direccionalidad causa-efecto entre los factores asociados y la no utilización de los servicios de salud.

Finalmente se puede concluir que tener una enfermedad mental y no ser tratado con respeto según el género predisponen a los miembros del colectivo LGBTI a no utilizar los servicios de salud. Contrario a esto, los factores que influyeron a utilizar los servicios de salud fueron haber expresado abiertamente su orientación sexual o estar afiliado en algún tipo de seguro de salud.

Contribuciones de los autores: LR y JVC concibieron y diseñaron el estudio, y analizaron los datos recolectados. LR, KLP, WRG y AJU recolectaron los datos. DQL analizó los datos y se encargó de la revisión crítica del manuscrito. Todos los autores participaron en la redacción, revisión y aprobación del manuscrito para su publicación, y se declaran responsables de su contenido.

Financiamiento: Autofinanciado.

Conflictos de interés: Los autores declaran no tener conflictos de interés.

Material suplementario: Disponible en la versión electrónica de la RPMESP. 


\section{REFERENCIAS BIBLIOGRÁFICAS}

1. Russell ST, Fish JN. Mental Health in Lesbian, Gay, Bisexual, and Transgender (LGBT) Youth. Annu Rev Clin Psychol. 2016;12:465-487. doi: 10.1146/annurev-clinpsy-021815-093153.

2. Zeeman L, Sherriff N, Browne K, McGlynn N, Mirandola M, Gios L, et al. A review of lesbian, gay, bisexual, trans and intersex (LGBTI) health and healthcare inequalities. Eur J Public Health. 2019;29(5):974-80. doi: 10.1093/eurpub/cky226.

3. Socías ME, Marshall BD, Arístegui I, Romero M, Cahn P, Kerr T, et al. Factors associated with healthcare avoidance among transgender women in Argentina. International Journal for Equity in Health. 2014;13(1):81. doi: 10.1186/s12939-014-0081-7.

4. Casey LS, Reisner SL, Findling MG, Blendon RJ, Benson JM, Sayde JM, et al. Discrimination in the United States: Experiences of lesbian, gay, bisexual, transgender, and queer Americans. Health Serv Res. 2019;54(S2):1454-66. doi: 10.1111/1475-6773.13229.

5. Lazo-Gonzales O, Alcalde-Rabanal J, Espinosa-Henao O. El sistema de salud en Perú: Situación y desafíos [Internet]. Lima: Colegio Médico del Perú; 2016. [citado el 19 de noviembre de 2020]. Disponible en: http://web2016.cmp.org.pe/wp-content/uploads/2016/12/libroSistemaSaludPeru-.pdf.

6. The Lancet Public Health. LGBT: the vital fight for the right to health. Lancet Public Health. 2019;4(3):e116. doi: 10.1016/S24682667(19)30025-8.

7. Kavanagh M, Baral S, Milanga M, Sugarman J. Biometrics and public health surveillance in criminalised and key populations: policy, ethics, and human rights considerations. Lancet HIV. 2018; 6(1):51-59. doi: 10.1016/S2352-3018(18)30243-1.

8. Informe LGBT 2018. Derecho a la igualdad de las personas LGBT en el Perú: Perspectivas jurídicas y políticas [Internet]. Lima: Promsex; 2018 [citado el 13 de agosto de 2020]. Disponible en: https://promsex.org/ publicaciones/informe-lgbt-2018-derecho-a-la-igualdad-de-las-personas-lgbt-en-el-peru-perspectivas-juridicas-y-politicas/

9. Paulino DB, Rasera EF, Teixeira FDB. Discourses on the healthcare of lesbian, gay, bisexual, and transgender (LGBT) people adopted by doctors working in Brazil's family health strategy. Interface Commun Heal Educ. 2019;23. doi: 10.1590/interface.180279.

10. Instituto Nacional de Estadística e Informática del Perú. Primera Encuesta Virtual para Personas LGBTI 2017 [Internet]. INEI; 2017. [citado el 19 de julio de 2020]. Disponible en: http://iinei.inei.gob.pe/ microdatos/index.htm.

11. Catalán Aguila M. Principales barreras de acceso a servicios de salud para personas lesbianas, gay y bisexuales. Cuad Méd Soc. 2018;58(2):43-7.

12. Sánchez-Torresa DA. Accesibilidad a los servicios de salud: debate teórico sobre determinantes e implicaciones en la política pública de salud. Rev Med Inst Mex Seguro Soc. 2017;55(1):82-9.

13. Tabaac AR, Solazzo AL, Gordon AR, Austin SB, Guss C, Charlton BM. Sexual orientation-related disparities in healthcare access in three cohorts of U.S. adults. Prevent Medicine. 2020;132:105999. doi: 10.1016/j.ypmed.2020.105999.

14. Rivera-Felix LM, Burgos-López NH, Gomez-Diaz JZ, Moquillaza-Alcántara $\mathrm{VH}$. Factores asociados al acceso a la atención prenatal en los hospitales de Huaral y Chancay, Perú. An Fac med. 2018; 79(2):131137. doi: 10.15381/anales.v79i2.14939.

15. Mantilla-Villabona LY, Maturana-Martinez DMA, Ospina-Galeano DC, Amado-Niño AM, Uribe Perez CJ. Barreras para la atención en salud durante el diagnóstico y tratamiento del cáncer gástrico. MedUNAB. 2017;19(3):211-220. doi: 10.29375/01237047.2262.
16. Andina. Minsa implementa enfoque de respeto de la población LGTBI en servicios de salud mental. [Internet]. [Citado el 11 de setiembre de 2020]. Disponible en: https://andina.pe/agencia/noticia-minsa-implementa-enfoque-respeto-de-poblacion-lgtbi-servicios-salud-mental-753436.aspx

17. Martos A, Wilson P, Meyer I. Lesbian, gay, bisexual, and transgender (LGBT) health services in the United States: Origins, evolution, and contemporarylandscape. PLoS One. 2017; 12(7):e0180544. doi: 10.1371/ journal.pone.0180544.

18. Trinh MH, Agenor M, Austin B, Jackson CL. Health and healthcare disparities among U.S. women and men at the intersection of sexual orientation and race/ethnicity: a nationally representative cross-sectional study. BMC Public Health. 2017. 17:964. doi: 10.1186/s12889-017-4937-9.

19. Salazar X, Villayzan J, Silva-Santisteban A, Cáceres C. La Situación de las Personas Trans en el Perú: Aspectos Sociales y Epidemiológicos [Internet]. Lima: Universidad Peruana Cayetano Heredia; 2010 [citado el 18 de febrero de 2020]. Disponible en: https://www.researchgate.net/ publication/245180632_La_Situacion_de_las_Personas_Trans_en_el_ Peru_Aspectos_Sociales_y_Epidemiologicos.

20. Claire Learmonth C, Viloria R, Lambert C, Goldhammer H, Keuroghlian A, Barriers to insurance coverage for transgender patients. Am J Obstet Gynecol. 2018;219(3):272. doi: 10.1016/j.ajog.2018.04.046.

21. Sattler F, Durrbaum T. Minority stress and mental health in lesbian, gay male, and bisexual youths: A meta-analysis. J LGBT Youth. 2020;17(3):2098-314. doi: 10.1080/19361653.2019.1586615.

22. Rinehart R, Rao D, Amico R, Ruiz E, Brandes E, Correa C, et al. Experienced HIV-Related Stigma and Psychological Distress in Peruvian Sexual and Gender Minorities: A Longitudinal Study to Explore Mediating Roles of Internalized HIV-Related Stigma and Coping Styles. AIDS Behav. 2019;23,661-674. doi: 10.1007/s10461-018-2348-2.

23. Verrelli S, White FA, Harvey LJ, Pulciani MR. Minority stress, social support, and the mental health of lesbian, gay, and bisexual Australians during the Australian Marriage Law Postal Survey. Aust Psychol. 2019;1-11. doi: 10.1111/ap.12380.

24. Juárez-Chávez E, Cooney E, Hidalgo A, Sánchez J, Poteat T. Violence Experiences in Childhood and Adolescence Among Gay Men and Transgender Women Living in Perú: A Qualitative Exploration. J Interpers Violence. 2018;886260518787811. doi: $10.1177 / 0886260518787811$.

25. Ferlatte O, Salway T, Rice S, Oliffe JL, Rich AJ, Knight R, et al. Perceived Barriers to Mental Health Services Among Canadian Sexual and Gender Minorities with Depression and at Risk of Suicide. Community Ment Health J. 2019;55(8):1313-21. doi: 10.1007/s10597-019-00445-1.

26. Huarcaya-Victoria J, Dávila-Palacios J, de la Cruz-Oré J. Relationship between the attitude towards homosexuality and religious attitude in physicians of a general hospital. An Fac med. 2018;79(2):138-143. doi: 10.15381/anales.v79i2.14940.

27. Pachankis JE, Goldfried MR. Clinical issues in working with lesbian, gay, and bisexual clients. Psychother Theory Res Pract. 2004;41:227-46 doi: 10.1037/2329-0382.1.S.45.

28. Baldwin A, Dodge B, Schick B, Sanders S, Fortenberry D. Sexual Minority Women's Satisfaction with Health Care Providers and State-level Structural Support: Investigating the Impact of Lesbian, Gay, Bisexual, and Transgender Nondiscrimination Legislation. Women's health issues. 2017;27(3):271-8. doi: 10.1016/j.whi.2017.01.004

29. Jaffee K, Shires D, Stroumsa D. Discrimination and Delayed Health Care Among Transgender Women and Men. Medical Care. 2016;54(11):101016. doi: 10.1097/MLR.0000000000000583.

30. Zeeman L, Aranda KA. Systematic Review of the Health and Healthcare Inequalities for People with Intersex Variance. Int J Environ Res Public Health. 2020;17(18):6533. doi: 10.3390/ijerph17186533. 


\section{ERRATA}

En el artículo "Factores asociados a la no utilización de servicios de salud en personas LGBTI de Perú", con número DOI: 10.17843/rpmesp.2021.382.6149, publicado en la Rev Peru Med Exp Salud Publica. 2021;38(2):240-7.

p. 244

Donde dice:

Tabla 1. Características generales de la población LGBTI estudiada

\begin{tabular}{|c|c|c|}
\hline Característica & $\mathbf{n}$ & $\%$ \\
\hline Edad & \multicolumn{2}{|c|}{$25(21-30)^{\mathrm{a}}$} \\
\hline \multicolumn{3}{|l|}{ Sexo registrado al nacer } \\
\hline Femenino & 2400 & 44,6 \\
\hline Masculino & 2984 & 55,4 \\
\hline \multicolumn{3}{|l|}{ Grado de instrucción } \\
\hline Sin estudios superiores & 751 & 14,0 \\
\hline Con estudios técnicos & 1089 & 20,3 \\
\hline Estudios universitarios & 3058 & 57,1 \\
\hline Estudios de posgrado & 461 & 8,6 \\
\hline \multicolumn{3}{|l|}{ Pareja } \\
\hline Sin pareja & 2777 & 52,2 \\
\hline Con pareja, sin convivencia & 1671 & 31,4 \\
\hline Con pareja, en convivencia & 764 & 14,4 \\
\hline Con más de una pareja & 105 & 2,0 \\
\hline \multicolumn{3}{|l|}{ Orientación sexual } \\
\hline Heterosexual & 119 & 2,2 \\
\hline Homosexual, gay & 2379 & 44,2 \\
\hline Homosexual, lesbiana & 989 & 18,4 \\
\hline Bisexual & 1366 & 25,4 \\
\hline Otro $^{\mathrm{b}}$ & 530 & 9,8 \\
\hline \multicolumn{3}{|l|}{ Intersexual } \\
\hline No & 4477 & 84,2 \\
\hline Sí & 233 & 4,38 \\
\hline No sabe & 607 & 11,42 \\
\hline \multicolumn{3}{|l|}{ Identidad de género } \\
\hline Persona transgénero $^{c}$ & 316 & 5,9 \\
\hline Persona cisgénero & 4540 & 85,2 \\
\hline Persona de queer género ${ }^{\mathrm{d}}$ & 474 & 8,9 \\
\hline
\end{tabular}

${ }^{\mathrm{a}}$ Mediana y rangos intercuartílicos; ${ }^{\mathrm{b}}$ pansexual, asexual, demisexual; ' transexual, transgénero, travesti; ${ }^{\mathrm{d}}$ personas de género no binario. 
Debe decir:

Tabla 1. Características generales de la población LGBTI estudiada.

\begin{tabular}{|c|c|c|}
\hline Característica & $\mathrm{n}^{\mathrm{a}}$ & $\%$ \\
\hline Edad & \multicolumn{2}{|c|}{$25(21-30)^{b}$} \\
\hline \multicolumn{3}{|l|}{ Sexo registrado al nacer } \\
\hline Femenino & 2400 & 44,6 \\
\hline Masculino & 2984 & 55,4 \\
\hline Datos perdidos & 2 & \\
\hline \multicolumn{3}{|l|}{ Grado de instrucción } \\
\hline Sin estudios superiores & 751 & 14 \\
\hline Con estudios técnicos & 1089 & 20,3 \\
\hline Estudios Universitarios & 3058 & 57,1 \\
\hline Estudios de posgrado & 461 & 8,6 \\
\hline Datos perdidos & 27 & \\
\hline \multicolumn{3}{|l|}{ Pareja } \\
\hline Sin pareja & 2777 & 52,2 \\
\hline Con pareja, sin convivencia & 1671 & 31,4 \\
\hline Con pareja, en convivencia & 764 & 14,4 \\
\hline Con más de una pareja & 105 & 2,0 \\
\hline Datos perdidos & 69 & \\
\hline \multicolumn{3}{|l|}{ Orientación sexual } \\
\hline Heterosexual & 119 & 2,2 \\
\hline Homosexual, gay & 2379 & 44,2 \\
\hline Homosexual, lesbiana & 989 & 18,4 \\
\hline Bisexual & 1366 & 25,4 \\
\hline Otro $^{c}$ & 530 & 9,8 \\
\hline Datos perdidos & 3 & \\
\hline \multicolumn{3}{|l|}{ Intersexual } \\
\hline No & 4477 & 84,2 \\
\hline Sí & 233 & 4,38 \\
\hline No sabe & 607 & 11,42 \\
\hline Datos perdidos & 69 & \\
\hline \multicolumn{3}{|l|}{ Identidad de género } \\
\hline Persona transgénero ${ }^{\mathrm{d}}$ & 316 & 5,9 \\
\hline Persona cisgénero & 4540 & 85,2 \\
\hline Persona de queer género ${ }^{e}$ & 474 & 8,9 \\
\hline Datos perdidos & 56 & \\
\hline
\end{tabular}

aNo todas las variables tienen la misma cantidad de observaciones, se expresan los datos perdidos en una fila adicional en cada categoría, no contabilizándose en las frecuencias relativas; ${ }^{\text {b }}$ mediana y rangos intercuartílicos; 'pansexual, asexual, demisexual; d transexual, transgénero; ${ }^{e}$ personas de género no binario

Rev Peru Med Exp Salud Publica. 2021;38(4):672-5. 


\section{p. 245}

Donde dice:

Tabla 2. Análisis bivariado y multivariado entre los factores asociados a la no utilización de servicios de salud en personas LGBTI en Perú

\begin{tabular}{|c|c|c|c|c|c|c|c|c|}
\hline \multirow{3}{*}{ Variable } & \multicolumn{8}{|c|}{ No utilización de servicios de salud } \\
\hline & \multicolumn{2}{|c|}{ Modelo crudo } & \multicolumn{2}{|c|}{ Modelo ajustado 1} & \multicolumn{2}{|c|}{ Modelo ajustado 2} & \multicolumn{2}{|c|}{ Modelo ajustado 3} \\
\hline & RPc (IC 95\%) & Valor de $\mathrm{p}$ & RPa (IC 95\%) & Valor de $\mathrm{p}$ & RPa (IC 95\%) & Valor de p & $\mathrm{RPa}(\mathrm{IC}$ 95\%) & Valor de $p$ \\
\hline Edad & $0,94(0,93-0,95)$ & $<0,001$ & $0,99(0,98-1,00)$ & 0,118 & $0,99(0,98-1,00)$ & 0,115 & $0,99(0,98-1,00)$ & 0,081 \\
\hline \multicolumn{9}{|l|}{ Sexo registrado al nacer } \\
\hline Femenino & Referencia & & Referencia & & Referencia & & Referencia & \\
\hline Masculino & $0,64(0,56-0,73)$ & $<0,001$ & $1,17(0,95-1,45)$ & 0,129 & $1,19(1,04-1,37)$ & 0,013 & $1,19(1,04-1,36)$ & 0,013 \\
\hline \multicolumn{9}{|c|}{ Expresa sin temor su orientación sexual o identidad de género } \\
\hline No & Referencia & & Referencia & & Referencia & & Referencia & \\
\hline Sí & $0,70(0,61-0,81)$ & $<0,001$ & $0,80(0,70-0,93)$ & 0,003 & $0,80(0,70-0,93)$ & 0,003 & $0,80(0,64-0,93)$ & 0,003 \\
\hline \multicolumn{9}{|c|}{ Le obligaron a someterse a pruebas de ITS o VIH } \\
\hline No & Referencia & & Referencia & & Referencia & & Referencia & \\
\hline Sí & $0,60(0,44-0,82)$ & $<0,001$ & $0,78(0,60-1,03)$ & 0,078 & $0,78(0,60-1,03)$ & 0,078 & $0,80(0,61-1,05)$ & 0,610 \\
\hline \multicolumn{9}{|l|}{ Nivel de estudios alcanzado } \\
\hline Sin estudios superiores & Referencia & & Referencia & & Referencia & & Referencia & \\
\hline Con estudios técnicos & $0,74(0,61-0,91)$ & 0,004 & $1,03(0,81-1,25)$ & 0,972 & $1,01(0,81-1,26)$ & 0,943 & $0,99(0,80-1,24)$ & 0,956 \\
\hline Estudios universitarios & $0,82(0,69-0,96)$ & 0,017 & $0,89(0,74-1,07)$ & 0,208 & $0,89(0,74-1,07)$ & 0,198 & $0,89(0,74-1,06)$ & 0,197 \\
\hline Estudios de posgrado & $0,31(0,21-0,46)$ & $<0,001$ & $0,50(0,33-0,76)$ & 0,001 & $0,49(0,32-0,75)$ & 0,001 & $0,49(0,34-0,75)$ & 0,001 \\
\hline \multicolumn{9}{|l|}{ Relación de pareja } \\
\hline Sin pareja & Referencia & & Referencia & & Referencia & & Referencia & \\
\hline Con pareja sin convivencia & $0,90(0,78-1,05)$ & 0,211 & $0,95(0,82-1,10)$ & 0,486 & $0,96(0,83-1,11)$ & 0,563 & $0,96(0,87-1,11)$ & 0,577 \\
\hline Con pareja en convivencia & $0,62(0,49-0,78)$ & $<0,001$ & $0,95(0,76-1,18)$ & 0,647 & $0,93(0,74-1,17)$ & 0,547 & $0,95(0,76-1,19)$ & 0,662 \\
\hline Más de una pareja & $0,65(0,36-1,15)$ & 0,141 & $1,17(0,62-2,18)$ & 0,630 & $1,36(0,75-2,45)$ & 0,314 & $1,20(0,65-2,22)$ & 0,560 \\
\hline \multicolumn{9}{|l|}{ Lugar de residencia } \\
\hline Provincia & Referencia & & Referencia & & Referencia & & Referencia & \\
\hline Lima & $0,84(0,73-0,97)$ & 0,021 & $0,98(0,95-1,02)$ & 0,378 & $0,98(0,95-1,02)$ & 0,399 & $0,98(0,95-1,02)$ & $<0,001$ \\
\hline \multicolumn{9}{|l|}{ Seguro de salud } \\
\hline No & Referencia & & Referencia & & Referencia & & Referencia & \\
\hline Sí & $0,51(0,44-0,58)$ & $<0,001$ & $0,75(0,66-0,85)$ & $<0,001$ & $0,75(0,66-0,86)$ & $<0,001$ & $0,75(0,66-0,85)$ & $<0,001$ \\
\hline \multicolumn{9}{|c|}{ Antecedentes de enfermedades crónicas no transmisibles } \\
\hline No & Referencia & & Referencia & & Referencia & & Referencia & \\
\hline Sí & $0,26(0,21-0,33)$ & $<0,001$ & $0,72(0,33-0,54)$ & $<0,001$ & $0,42(0,33-054)$ & $<0,001$ & $0,41(0,33-0,54)$ & $<0,001$ \\
\hline \multicolumn{9}{|c|}{ Antecedentes de enfermedades infectocontagiosas } \\
\hline No & Referencia & & Referencia & & Referencia & & Referencia & \\
\hline Sí & $0,10(0,07-0,15)$ & $<0,001$ & $0,18(0,11-0,29)$ & $<0,001$ & $0,18(0,12-0,29)$ & $<0,001$ & $0,18(0,11-0,29)$ & $<0,001$ \\
\hline \multicolumn{9}{|c|}{ Antecedentes de problemas de salud mental } \\
\hline No & Referencia & & Referencia & & Referencia & & Referencia & \\
\hline Sí & $8,48(6,84-10,52)$ & $<0,001$ & $4,78(3,71-6,15)$ & $<0,001$ & $4,85(3,77-6,25)$ & $<0,001$ & $4,74(3,68-6,10)$ & $<0,001$ \\
\hline Respeto por su identidad de gen & & & & & & & & \\
\hline No & Referencia & & Referencia & & Referencia & & Referencia & \\
\hline Sí & $1,25(1,05-1,50)$ & 0,014 & $1,26(1,06-1,50)$ & 0,010 & $1,29(0,93-1,23)$ & 0,333 & $1,26(1,06-1,49)$ & 0,008 \\
\hline Cambio de apariencia & & & & & & & & \\
\hline No & Referencia & & Referencia & & Referencia & & Referencia & \\
\hline Sí & $1,34(1,16-1,56)$ & $<0,001$ & $1,08(0,94-1,24)$ & 0,288 & $1,08(0,93-1,24)$ & 0,333 & $1,08(0,94-1,24)$ & 0,285 \\
\hline Orientación sexual & & & & & & & & \\
\hline Heterosexual & Referencia & & Referencia & & - & - & - & - \\
\hline Homosexual, gay & $0,67(0,45-0,99)$ & 0,049 & $1,11(0,72-1,72)$ & 0,636 & - & - & - & - \\
\hline Homosexual, lesbiana & $0,89(0,59-1,35)$ & 0,595 & $1,08(0,70-1,69)$ & 0,722 & - & - & - & - \\
\hline Bisexual & $1,17(0,78-1,75)$ & 0,444 & $1,14(0,75-1,75)$ & 0,540 & - & - & - & - \\
\hline Otros $^{\mathrm{a}}$ & $1,31(0,87-1,99)$ & 0,199 & $1,09(0,70-1,71)$ & 0,693 & - & - & - & - \\
\hline Identidad de género & & & & & & & & \\
\hline Cisgénero & Referencia & & - & - & Referencia & & - & - \\
\hline Transgénero & $1,17(0,801,71)$ & 0,409 & - & - & $0,91(0,69-1,20)$ & 0,515 & - & - \\
\hline Queer género & $1,30(1,051,61)$ & 0,014 & - & - & $1,07(0,88-1,12)$ & 0,496 & - & - \\
\hline Intersexualidad & & & & & & & & \\
\hline No & Referencia & & - & - & - & - & Referencia & \\
\hline Sí & $0,57(0,38-, 086)$ & 0,007 & - & - & - & - & $0,64(0,41-0,99)$ & 0,045 \\
\hline No sabe & $1,25(1,05-1,48)$ & 0,013 & - & - & - & - & $1,14(0,94-1,37)$ & 0,175 \\
\hline
\end{tabular}

Modelo 1. Incluye la variable orientación sexual. Modelo 2. Incluye la variable identidad de género. Modelo 3. Incluye la variable intersexualidad. a demisexual, sapiosexual. RPa: razón de prevalencia ajustada; IC $95 \%$ : intervalo de confianza al 95\%. 
Debe decir:

Tabla 2. Análisis bivariado y multivariado entre los factores asociados a la no utilización de servicios de salud en personas LGBTI en Perú.

\begin{tabular}{|c|c|c|c|c|c|c|c|c|}
\hline \multirow{3}{*}{ Variable } & \multicolumn{8}{|c|}{ No utilización de servicios de salud } \\
\hline & \multicolumn{2}{|c|}{ Modelo crudo } & \multicolumn{2}{|c|}{ Modelo ajustado 1} & \multicolumn{2}{|c|}{ Modelo ajustado 2} & \multicolumn{2}{|c|}{ Modelo ajustado 3} \\
\hline & RPc (IC 95\%) & Valor de p & RPa (IC 95\%) & Valor de p & RPa (IC 95\%) & Valor de p & RPa (IC 95\%) & Valor de p \\
\hline Edad & $0,94(0,93-0,95)$ & $<0,001$ & $0,99(0,98-1,00)$ & 0,118 & $0,99(0,98-1,00)$ & 0,115 & $0,99(0,98-1,00)$ & 0,081 \\
\hline \multicolumn{9}{|l|}{ Sexo registrado al nacer } \\
\hline Femenino & Referencia & & Referencia & & Referencia & & Referencia & \\
\hline Masculino & $0,64(0,56-0,73)$ & $<0,001$ & $1,17(0,95-1,45)$ & 0,129 & $1,19(1,04-1,37)$ & 0,013 & $1,19(1,04-1,36)$ & 0,013 \\
\hline \multicolumn{9}{|c|}{ Expresa sin temor su orientación sexual o identidad de género } \\
\hline No & Referencia & & Referencia & & Referencia & & Referencia & \\
\hline Sí & $0,70(0,61-0,81)$ & $<0,001$ & $0,80(0,70-0,93)$ & 0,003 & $0,80(0,70-0,93)$ & 0,003 & $0,80(0,64-0,93)$ & 0,003 \\
\hline \multicolumn{9}{|c|}{ Le obligaron a someterse a pruebas de ITS o VIH } \\
\hline No & Referencia & & Referencia & & Referencia & & Referencia & \\
\hline Sí & $0,60(0,44-0,82)$ & $<0,001$ & $0,78(0,60-1,03)$ & 0,078 & $0,78(0,60-1,03)$ & 0,078 & $0,80(0,61-1,05)$ & 0,610 \\
\hline \multicolumn{9}{|l|}{ Nivel de estudios alcanzado } \\
\hline Sin estudios superiores & Referencia & & Referencia & & Referencia & & Referencia & \\
\hline Con estudios técnicos & $0,74(0,61-0,91)$ & 0,004 & $1,03(0,81-1,25)$ & 0,972 & $1,01(0,81-1,26)$ & 0,943 & $0,99(0,80-1,24)$ & 0,956 \\
\hline Estudios universitarios & $0,82(0,69-0,96)$ & 0,017 & $0,89(0,74-1,07)$ & 0,208 & $0,89(0,74-1,07)$ & 0,198 & $0,89(0,74-1,06)$ & 0,197 \\
\hline Estudios de posgrado & $0,31(0,21-0,46)$ & $<0,001$ & $0,50(0,33-0,76)$ & 0,001 & $0,49(0,32-0,75)$ & 0,001 & $0,49(0,34-0,75)$ & 0,001 \\
\hline \multicolumn{9}{|l|}{ Relación de pareja } \\
\hline Sin pareja & Referencia & & Referencia & & Referencia & & Referencia & \\
\hline Con pareja sin convivencia & $0,90(0,78-1,05)$ & 0,211 & $0,95(0,82-1,10)$ & 0,486 & $0,96(0,83-1,11)$ & 0,563 & $0,96(0,87-1,11)$ & 0,577 \\
\hline Con pareja en convivencia & $0,62(0,49-0,78)$ & $<0,001$ & $0,95(0,76-1,18)$ & 0,647 & $0,93(0,74-1,17)$ & 0,547 & $0,95(0,76-1,19)$ & 0,662 \\
\hline Más de una pareja & $0,65(0,36-1,15)$ & 0,141 & $1,17(0,62-2,18)$ & 0,630 & $1,36(0,75-2,45)$ & 0,314 & $1,20(0,65-2,22)$ & 0,560 \\
\hline \multicolumn{9}{|l|}{ Lugar de residencia } \\
\hline Provincia & Referencia & & Referencia & & Referencia & & Referencia & \\
\hline Lima & $0,84(0,73-0,97)$ & 0,021 & $0,98(0,95-1,02)$ & 0,378 & $0,98(0,95-1,02)$ & 0,399 & $0,98(0,95-1,02)$ & 0,375 \\
\hline \multicolumn{9}{|l|}{ Seguro de salud } \\
\hline No & Referencia & & Referencia & & Referencia & & Referencia & \\
\hline Sí & $0,51(0,44-0,58)$ & $<0,001$ & $0,75(0,66-0,85)$ & $<0,001$ & $0,75(0,66-0,86)$ & $<0,001$ & $0,75(0,66-0,85)$ & $<0,001$ \\
\hline \multicolumn{9}{|c|}{ Antecedentes de enfermedades crónicas no transmisibles } \\
\hline No & Referencia & & Referencia & & Referencia & & Referencia & \\
\hline Sí & $0,26(0,21-0,33)$ & $<0,001$ & $0,72(0,33-0,54)$ & $<0,001$ & $0,42(0,33-054)$ & $<0,001$ & $0,41(0,33-0,54)$ & $<0,001$ \\
\hline \multicolumn{9}{|c|}{ Antecedentes de enfermedades infectocontagiosas } \\
\hline No & Referencia & & Referencia & & Referencia & & Referencia & \\
\hline Sí & $0,10(0,07-0,15)$ & $<0,001$ & $0,18(0,11-0,29)$ & $<0,001$ & $0,18(0,12-0,29)$ & $<0,001$ & $0,18(0,11-0,29)$ & $<0,001$ \\
\hline \multicolumn{9}{|c|}{ Antecedentes de problemas de salud mental } \\
\hline No & Referencia & & Referencia & & Referencia & & Referencia & \\
\hline Sí & $8,48(6,84-10,52)$ & $<0,001$ & $4,78(3,71-6,15)$ & $<0,001$ & $4,85(3,77-6,25)$ & $<0,001$ & $4,74(3,68-6,10)$ & $<0,001$ \\
\hline Respeto por su identidad de gen & & & & & & & & \\
\hline No & Referencia & & Referencia & & Referencia & & Referencia & \\
\hline Sí & $1,25(1,05-1,50)$ & 0,014 & $1,26(1,06-1,50)$ & 0,010 & $1,29(0,93-1,23)$ & 0,333 & $1,26(1,06-1,49)$ & 0,008 \\
\hline Cambio de apariencia & & & & & & & & \\
\hline No & Referencia & & Referencia & & Referencia & & Referencia & \\
\hline Sí & $1,34(1,16-1,56)$ & $<0,001$ & $1,08(0,94-1,24)$ & 0,288 & $1,08(0,93-1,24)$ & 0,333 & $1,08(0,94-1,24)$ & 0,285 \\
\hline Orientación sexual & & & & & & & & \\
\hline Heterosexual & Referencia & & Referencia & & - & - & - & - \\
\hline Homosexual, gay & $0,67(0,45-0,99)$ & 0,049 & $1,11(0,72-1,72)$ & 0,636 & - & - & - & - \\
\hline Homosexual, lesbiana & $0,89(0,59-1,35)$ & 0,595 & $1,08(0,70-1,69)$ & 0,722 & - & - & - & - \\
\hline Bisexual & $1,17(0,78-1,75)$ & 0,444 & $1,14(0,75-1,75)$ & 0,540 & - & - & - & - \\
\hline Otros $^{\mathrm{a}}$ & $1,31(0,87-1,99)$ & 0,199 & $1,09(0,70-1,71)$ & 0,693 & - & - & - & - \\
\hline Identidad de género & & & & & & & & \\
\hline Cisgénero & Referencia & & - & - & Referencia & & - & - \\
\hline Transgénero & $1,17(0,801,71)$ & 0,409 & - & - & $0,91(0,69-1,20)$ & 0,515 & - & - \\
\hline Queer género & $1,30(1,051,61)$ & 0,014 & - & - & $1,07(0,88-1,12)$ & 0,496 & - & - \\
\hline Intersexualidad & & & & & & & & \\
\hline No & Referencia & & - & - & - & - & Referencia & \\
\hline Sí & $0,57(0,38-, 086)$ & 0,007 & - & - & - & - & $0,64(0,41-0,99)$ & 0,045 \\
\hline No sabe & $1,25(1,05-1,48)$ & 0,013 & - & - & - & - & $1,14(0,94-1,37)$ & 0,175 \\
\hline
\end{tabular}

Modelo 1. Incluye la variable orientación sexual. Modelo 2. Incluye la variable identidad de género. Modelo 3. Incluye la variable intersexualidad. a demisexual, sapiosexual. RPa: razón de prevalencia ajustada; IC $95 \%$ : intervalo de confianza al 95\%.

Rev Peru Med Exp Salud Publica. 2021;38(4):672-5. 